\title{
Plane waves in a relativistic homogeneous and isotropic elastic continuum
}

\author{
Vratko Polák* and Vladimír Balek ${ }^{\dagger}$ \\ Department of Theoretical Physics, Comenius University, Mlynská dolina \\ 84248 Bratislava, Slovakia
}

July 20, 2018

\begin{abstract}
Propagation of gravitational and acoustic plane waves in a flat universe filled with a general relativistic, homogeneous and isotropic, spatially flat continuum is studied. The continuum is described by analogues of nonrelativistic characteristics, namely energy per particle, pressure and Lame coefficients, and considered in the comoving proper-time gauge. For all modes with the given wave covector, differential equations governing the time dependence of the amplitudes are derived. In particular, longitudinal acoustic waves are described, in analogy with the nonrelativistic theory, by two coupled first-order equations. As an example, plane waves in a stiff ultrarigid continuum are considered.
\end{abstract}

\section{Introduction}

Elasticity, as a theory of matter in form of an (ideal, dissipationless) elastic continuum, has many applications in everyday life; but in areas where relativistic effects are important, applications are much more sparse. Nevertheless, the presence of a solid crust in neutron stars has motivated the development of relasticity (short name for relativistic elasticity, from [1]). Among early works on the topic, 2] appears to be the most successful.

Since then, fundamental concepts of relasticity are known, but the terminology, notation and formalism are far from being stabilized. Different authors start from different notions of nonrelativistic elasticity, and use different coordinates, depending on what they find most natural and/or appropriate for the problem they study. This paper is no exception. We deal with a particular problem, thus we consider particular notions to be basic, develop particular formalism and perform computations in particular coordinates.

The motivation of this paper is that, as long as elastic continuum can be included into the framework of general relativity, we should be able to describe how nonrelativistic elastic phenomena and Einstein's gravitational phenomena affect each other.

\footnotetext{
*e-mail address: polak@fmph.uniba.sk
}

${ }^{\dagger}$ e-mail address: balek@fmph.uniba.sk 
As a nonrelativistic effect to start with, we have chosen the propagation of acoustic waves; specifically, its simplest case, the propagation of plane waves in a homogeneous and isotropic continuum. For details, see [3].

In general relativity, the effects described by the theory also include plane waves in a homogeneous and isotropic background, namely gravitational waves in a flat expanding universe.

The first goal of this paper is to derive equations of propagation of both acoustic and gravitational (weak) waves in a homogeneous and isotropic, spatially flat, but otherwise completely general continuum. Since less specific equations for general weak waves were formulated, and the correctness of their nonrelativistic limit was proven in [4], we do not address the problem of nonrelativistic limit here.

The second goal of this paper is to show how to use the theory of relasticity when solving a particular problem, if simplicity and clarity are considered more important than elegance and universality. This goal includes introducing such formalism, and using such coordinates, that the result will be obtained in the easiest possible way, even if that formalism and those coordinates will not prove useful in other relasticity problems.

This paper is motivated neither by astrophysics nor by cosmology, although its results can be applied within the latter, when considering a solid dark matter component as in [5]. Therefore, when describing our results, we remind the reader also of the terminology used in the theory of cosmological perturbations, see for example [6]. Our notations in relasticity are chosen to be compatible with [7], and our notations in differential geometry to be compatible with [8].

The paper is organized as follows. In section 2 we introduce basic concepts of relasticity. In section 3 we develop a theory of plane waves as linear perturbations imposed on the background solution. We derive equations for the background as well as for the perturbations, identify modes that correspond to gravitational and acoustic (transversal and longitudinal) waves, and propose a gauge invariant description of longitudinal acoustic waves. For better understanding of our formalism, in section 4 we write down equations for plane waves in a particular continuum, namely the stiff ultrarigid continuum introduced in [9]. In section 5 we discuss the results.

\section{Relasticity}

Relasticity is a theory that describes elastic continua within Einstein's theory of gravity. In the simplest case there is just one continuum, and it extends over all spacetime. The spacetime is, of course, a 4-dimensional manifold, equipped with a metric $g$ that is regarded as a dynamical field satisfying the Einstein equations.

Properties of the continuum are defined on the material manifold, that is, on a 3-dimensional manifold whose points represent particles of which the continuum consists. The way the continuum is distributed in spacetime is described by the mapping $f$ that maps spacetime on the material manifold, telling us which particle is in the particular worldpoint. It is called material mapping and considered a dynamical field on the spacetime, too. Contravariant metric in any worldpoint can be push-forwarded by the material mapping, providing us with a symmetric, double contravariant tensor on the material manifold. This tensor can be interpreted as the strain tensor.

The only characteristic of continuum that influences its dynamics is the state function $\varrho$. It is a scalar function with two arguments, the point of the material manifold and the strain tensor 
at that point. By definition, it is the energy density in the rest frame of the given particle, in the continuum whose deformation is described by the given strain tensor.

The dynamics is determined by the action $S=\int(-\rho+R) \omega_{g}$, where $R$ is the scalar curvature, $\rho$ is the energy density given by the state function and $\omega_{g}$ is the 4 -form of volume defined by the metric $g$ (for details, see [8]). We use a system of units in which the factor in front of $R$ involving gravitational constant is absent. In these units, the Einstein equations assume the form $2 G_{\mu \nu}=T_{\mu \nu}$.

The principle of extremal action provides us with the equations of motion. Variation of the action with respect to the metric leads to the Einstein equations with an explicit formula for the energy-momentum tensor (to be given in the next section). Variation of the action with respect to the material mapping yields equations of motion of the continuum (also written in the next section). As a matter of fact, Einstein equations together with the Bianchi identity usually carry enough information to describe the dynamics completely; nevertheless, it is useful to write down the equations governing the dynamics of the source explicitly.

The action, and therefore also equations of motion, is covariant with respect to the change of coordinates in the spacetime, and also in the material manifold. This is quite a large gauge freedom. This freedom, however, can be used for fixing coordinate-dependent expressions in such a way that they become simpler (although less general). In this paper, we fix the gauge almost completely in a way we find most suitable for our problem.

\section{Plane waves}

We will investigate the propagation of weak waves, which can be regarded as linear perturbations to a certain background solution.

\subsection{Description of the continuum; the background solution}

For simplicity, we choose the elastic continuum to be homogeneous, isotropic and flat, which means that there exists a set of coordinates $X^{A}$ in the material manifold (where the material index $A$, as well as any other upper case Latin index, runs from 1 to 3 ) such that the state function is invariant with respect to Euclidean translations and rotations of $X^{A}$.

The strain tensor has coordinate components (with respect to $X^{A}$ ) which can be treated as coordinates on the space of double contravariant tensors on the material manifold. These components will be denoted by $H^{A B}$. The true strain tensors are always symmetric, but for the purposes of differentiation we can extend the state function also to nonsymmetric tensors. This gives correct results if we let $\varrho$ depend only on the symmetric part of $H^{A B}$, or if we symmetrize, when differentiating with respect to $H^{A B}$ more than once, the derivatives with respect to all $H^{A B}$ 's but the last (and of course, insert symmetric values into the resulting expressions). We will adopt the latter procedure. In general, $\varrho$ can be differentiated also with respect to $X^{A}$. However, the invariance with respect to translations implies $\partial \varrho / \partial X^{A}=0$, therefore only the derivatives of $\varrho$ with respect to (the nine components of) $H^{A B}$ enter the equations.

Having fixed the coordinates $X^{A}$ on the material manifold, we can express the material mapping $f$ through the coordinate component functions $f^{A}$. Together with arbitrary coordinates $x^{\mu}$ in the spacetime (where the spacetime index $\mu$, as well as any other Greek index, runs from 
0 to 3 ; we will also use lower case Latin letters to denote space indices running from 1 to 3 ), we now have everything ready for the variation of the action with respect to $f^{A}$. This variation leads to the equations of motion of a homogeneous isotropic flat continuum

$$
\left(\frac{\partial \varrho}{\partial H^{A B}} g^{\mu \nu}+2 \frac{\partial^{2} \varrho}{\partial H^{A C} \partial H^{B D}} g^{\mu \lambda} g^{\nu \kappa} f_{, \lambda}^{C} f_{, \kappa}^{D}\right) f_{, \nu ; \mu}^{B}=0
$$

By varying the material part of the action with respect to $g_{\mu \nu}$, we obtain a formula for the energy-momentum tensor,

$$
T_{\mu \nu}=2 \frac{\partial \varrho}{\partial H^{A B}} f_{, \mu}^{A} f_{, \nu}^{B}-\varrho g_{\mu \nu}
$$

Having completed a general description of the continuum under consideration, we can proceed to further simplifications. The gauge freedom in spacetime coordinates can be reduced by requiring that $f^{A}(x)=x^{A}\left(x^{A}\right.$ are comoving coordinates), after which there remains only one free coordinate $x^{0}$. Further restriction will be $g_{00}=-1$, which implies that $x^{0}$ equals the proper time $t$ of the particles of the continuum. The only freedom left consists in the choice of the hypersurface $x^{0}=0$.

Now we can specify the background. We assume that it is, just like the continuum, homogeneous, isotropic and flat, that is, equipped with a flat Robertson-Walker metric $g=$ $-d t \otimes d t+a^{2}\left(d x^{1} \otimes d x^{1}+d x^{2} \otimes d x^{2}+d x^{3} \otimes d x^{3}\right)$, where the scale parameter $a$ depends only on $t$. The symmetry allowed us to choose the zero time hypersurface to be orthogonal to the worldlines of all particles. Instead of the scale parameter, it turns out to be more convenient to use its $\log$ arithm $z=\ln a$. Then we have $g_{00}=-1, g_{0 i}=0, g_{i j}=e^{2 z} \delta_{i j}$, and we can compute the Einstein tensor to obtain $2 G_{00}=6 \dot{z}^{2}, 2 G_{0 i}=0,2 G_{i j}=e^{2 z}\left(-4 \ddot{z}-6 \dot{z}^{2}\right) \delta_{i j}$, where the overdot means differentiation with respect to $t$. The energy-momentum tensor simplifies to $T_{00}=\varrho$, $T_{0 i}=0, T_{i j}=2 \partial \varrho / \partial H^{i j}-e^{2 z} \varrho \delta_{i j}$. Equations of motion of the continuum hold identically.

The strain tensor of the background solution is $H^{A B}=e^{-2 z} \delta^{A B}$, and the invariance of $\varrho$ with respect to rotations ensures that the derivatives of $\varrho$ with respect to $H^{A B}$, evaluated for the background solution, are proportional to $\delta_{A B}$ (the first derivative) or equal to linear combinations of the products of $\delta_{A B}$ (the higher derivatives). Here, $\delta$ is in fact the Euclidean metric of the material manifold, but in our coordinates we can use notation reminding us that $\delta_{A B}$ and $\delta^{A B}$ are unit matrices. This enables us to employ an extended version of summation convention, for example $g^{A A}=g^{A B} \delta_{A B}$ and $g_{A A}=g_{A B} \delta^{A B}$. Since we have, in fact, identified the spatial coordinates with the material coordinates, we can pull-back $\delta$ to obtain the unit matrices $\delta_{i j}$ and $\delta^{i j}$. The first matrix was already used in the formulas for $2 G_{i j}$ and $T_{i j}$.

As the next step, let us introduce material characteristics to appear in the equations for the background, as well as in the equations for perturbations. First, Einstein's cosmological constant can be treated as the vacuum energy density $\rho_{0}$ contributing a constant term to $\varrho$. After separating out this term, we can write the remaining energy density as a product of the particle density $n$ and the energy per particle $\varepsilon$. In this way we obtain the general formula $\varrho=\rho_{0}+n \varepsilon$, which can be written for the background metric as $\varrho=\rho_{0}+e^{-3 z} \varepsilon$. Note that the separation of the vacuum energy density is in a sense artificial. We could choose any other value of $\rho_{0}$ and change $\varepsilon$ in such a way that $\varrho$ will be the same. Nevertheless, the introduction of $\rho_{0}$ is useful since it provides us with a simpler formula for the quantity $\varepsilon$ than we would obtain if we put simply $\varrho=n \varepsilon$. 
In the nonrelativistic theory, when a homogeneous and isotropic continuum is deformed in a homogeneous and isotropic way, its stress tensor is given by one scalar quantity - the pressure $\sigma$; and when it is relaxed, its elastic properties are determined by two scalar quantities - Lame coefficients $\mu$ and $\lambda$ (see, for example, [3]). Analogically, for a general relativistic homogeneous and isotropic continuum we define the scalar $\sigma$ by the relation

$$
2 \frac{\partial \varepsilon}{\partial H^{A B}}=e^{2 z} \sigma \delta_{A B}
$$

and the scalars $\mu$ and $\lambda$ by the relation

$$
2\left(\frac{\partial^{2} \varepsilon}{\partial H^{A B} \partial H^{C D}}+\frac{\partial^{2} \varepsilon}{\partial H^{A B} \partial H^{D C}}\right)=e^{4 z}\left[\lambda \delta_{A B} \delta_{C D}+\mu\left(\delta_{A C} \delta_{B D}+\delta_{A D} \delta_{B C}\right)\right] .
$$

With all this new scalars, the derivatives of $\varrho$ evaluated for the background metric can be written as

$$
2 \frac{\partial \varrho}{\partial H^{A B}}=e^{-z}(\varepsilon+\sigma) \delta_{A B}
$$

and

$$
2\left(\frac{\partial^{2} \varrho}{\partial H^{A B} \partial H^{C D}}+\frac{\partial^{2} \varrho}{\partial H^{A B} \partial H^{D C}}\right)=e^{z}\left[(\lambda+2 \sigma+\varepsilon) \delta_{A B} \delta_{C D}+(\mu-\varepsilon)\left(\delta_{A C} \delta_{B D}+\delta_{A D} \delta_{B C}\right)\right] .
$$

The scalars $\varepsilon, \sigma, \mu$ and $\lambda$ as functions of $z$ are not independent, because the derivative with respect to $z$ corresponds to the derivative with respect to $H^{A B}$. The relations between these scalars are $d \varepsilon / d z=-3 \sigma$ and $d \sigma / d z=-(2 \sigma+3 \lambda+2 \mu)$.

Now we are ready to write down the equations for the background. $T_{00}$ becomes $\rho_{0}+e^{-3 z} \varepsilon$, which gives $6 \dot{z}^{2}=\rho_{0}+e^{-3 z} \varepsilon$, and $T_{i j}$ becomes $e^{-z} \sigma \delta_{i j}-e^{2 z} \rho_{0} \delta_{i j}$, which gives, when combined with the equation for $\dot{z}^{2},-4 \ddot{z}=e^{-3 z}(\varepsilon+\sigma)$. If we take into account the identity for $d \varepsilon / d z$, we can see that the latter equation is nothing but the time derivative of the former equation. (At least, this holds if $\dot{z} \neq 0$, which we will assume in what follows in order to avoid singularities in our expressions. By the same reason, we will assume $\varepsilon+\sigma \neq 0$.) Thus, the background is a flat Robertson-Walker metric satisfying $6 \dot{z}^{2}=\rho_{0}+e^{-3 z} \varepsilon$ and $d \varepsilon / d z=-3 \sigma$; in other words, a flat universe evolving as if it contained, in addition to the dark energy with the density $\varrho_{0}$, an ideal fluid with the density $e^{-3 z} \varepsilon$ and pressure $e^{-3 z} \sigma$.

\subsection{Linear perturbations}

Having fixed the background solution we can start to inspect linear perturbations. Denote perturbations of the fields $f$ and $g$ by $\delta f$ and $\delta g$. Of course, not every perturbation is permitted by the dynamics, but on the other hand, some perturbations correspond merely to coordinate changes and are not of physical interest.

First, we reduce the gauge degrees of freedom in a way analogical to how we fixed the coordinates in the background solution. We require that for the perturbed solutions the spacetime coordinates are comoving, too, which implies $\delta f^{A}=0$. We want also the $x^{0}$ coordinate to measure the proper time of the particles $\left(x^{0}=t\right)$, which implies $\delta g_{00}=0$. This leaves us with the freedom of choosing the zero time hypersurface. But, unlike the background, the perturbed metric is generally not symmetric enough to allow us to pick a certain nice hypersurface (like the 
hypersurface orthogonal to the worldlines of all particles). Therefore we proceed further with this last ambiguity left.

Before writing down the linearized dynamical equations, we can take advantage of the fact that the linearized equations are (of course) linear. Therefore their solutions form a linear space (they satisfy the superposition principle), and can be complexified. The complex solutions have no direct physical meaning, but they can simplify the upcoming expressions a bit.

Since the solutions form a linear space, it is sufficient to investigate some basis of them to obtain the complete picture. A nice ansatz for such basis follows from the translation symmetry of the continuum. This symmetry ensures that there are solutions of the form of (complex) plane waves, that is, depending on the space coordinates only through the multiplication factor $\exp \left(i k_{A} x^{A}\right)$, where $k$ is a constant real wave covector on the material manifold. When dealing with just one plane wave, we can always utilize the rotational invariance of the background solution to rotate coordinates in such a way that $x^{1}$ becomes parallel to $k$, which means that the coordinate components of $k$ become $(k, 0,0)$ (the first $k$ is a covector, the second $k$ is a real number).

If we find all plane wave solutions, we can use them to construct any solution of the form of a (space-) tempered distribution. This means that we actually do not have a basis, because we miss the solutions that grow faster than polynomially with the space distance, as is the case for surface waves. We duly restrict our attention to the space-tempered distributions, referring to the fact that because of causality such solutions are sufficient for describing linearized dynamics in a bounded region of spacetime.

Plane waves provide a significant simplification of our problem since the space derivatives acting on the perturbations become just powers of $k$. In further computations, some typical combinations of derivatives with respect to time or space coordinates occur repeatedly, so it is convenient not to use $\delta g$ directly, but to replace it by more complicated expressions in order to obtain simpler looking results. For a plane wave with the wave covector $k$ already rotated into the $x^{1}$ direction we define a tensorial-looking, time-dependent quantity $h$ by putting

$$
\begin{gathered}
\delta g_{0 i}(x)=-i k h_{0 i}(t) e^{i k x^{1}}, \\
\delta g_{i j}(x)=e^{2 z(t)} h_{i j}(t) e^{i k x^{1}} .
\end{gathered}
$$

Now, everything is ready for a straightforward but lengthy variation of dynamical equations. We will write down just the final expressions. Variation of the energy-momentum tensor gives

$$
\begin{gathered}
\delta T_{00}=-\frac{1}{2} e^{-3 z}(\varepsilon+\sigma) h_{k k}, \\
\delta T_{0 i}=i k \rho_{0} h_{0 i}+i k e^{-3 z} \varepsilon h_{0 i}, \\
\delta T_{i j}=-\frac{1}{2} e^{-z}(\lambda+\sigma) \delta_{i j} h_{k k}-e^{-z} \mu h_{i j}-e^{2 z} \rho_{0} h_{i j} ;
\end{gathered}
$$

variation of the Einstein tensor gives

$$
\begin{gathered}
2 \delta G_{00}=2 \dot{z} \dot{h}_{k k}-4 e^{-2 z} k^{2} \dot{z} h_{01}+e^{-2 z} k^{2}\left(h_{k k}-h_{11}\right), \\
2 \delta G_{01}=i k \dot{h}_{11}-i k \dot{h}_{k k}+i k\left(4 \ddot{z}+6 \dot{z}^{2}\right) h_{01},
\end{gathered}
$$




$$
\begin{gathered}
2 \delta G_{0 \alpha}=i k \dot{h}_{1 \alpha}+i k\left(4 \ddot{z}+6 \dot{z}^{2}\right) h_{0 \alpha}-i k e^{-2 z} k^{2} h_{0 \alpha}, \\
2 \delta G_{11}=e^{2 z}\left[\ddot{h}_{11}+3 \dot{z} \dot{h}_{11}-\left(4 \ddot{z}+6 \dot{z}^{2}\right) h_{11}-\ddot{h}_{k k}-3 \dot{z} \dot{h}_{k k}\right], \\
2 \delta G_{1 \alpha}=e^{2 z}\left[\ddot{h}_{1 \alpha}+3 \dot{z} \dot{h}_{1 \alpha}-\left(4 \ddot{z}+6 \dot{z}^{2}\right) h_{1 \alpha}\right]-k^{2} \dot{h}_{0 \alpha}-k^{2} \dot{z} h_{0 \alpha}, \\
2 \delta G_{22}=e^{2 z}\left[\ddot{h}_{22}+3 \dot{z} \dot{h}_{22}-\left(4 \ddot{z}+6 \dot{z}^{2}\right) h_{22}-\ddot{h}_{k k}-3 \dot{z} \dot{h}_{k k}\right]+2 k^{2} \dot{h}_{01}+2 k^{2} \dot{z} h_{01}+k^{2}\left(h_{11}+h_{22}-h_{k k}\right), \\
2 \delta G_{33}=e^{2 z}\left[\ddot{h}_{33}+3 \dot{z} \dot{h}_{33}-\left(4 \ddot{z}+6 \dot{z}^{2}\right) h_{33}-\ddot{h}_{k k}-3 \dot{z} \dot{h}_{k k}\right]+2 k^{2} \dot{h}_{01}+2 k^{2} \dot{z} h_{01}+k^{2}\left(h_{11}+h_{33}-h_{k k}\right), \\
2 \delta G_{23}=e^{2 z}\left[\ddot{h}_{23}+3 \dot{z} \dot{h}_{23}-\left(4 \ddot{z}+6 \dot{z}^{2}\right) h_{23}\right]+k^{2} h_{23} ;
\end{gathered}
$$

and variation of the equation of motion of the continuum gives

$$
\begin{gathered}
(\varepsilon+\sigma) \dot{h}_{01}=(2 \mu+3 \lambda+5 \sigma) \dot{z} h_{01}-(\mu+\sigma) h_{11}-\frac{1}{2}(\lambda+\sigma) h_{k k} \\
(\varepsilon+\sigma) \dot{h}_{0 \alpha}=(2 \mu+3 \lambda+5 \sigma) \dot{z} h_{0 \alpha}-(\mu+\sigma) h_{1 \alpha} .
\end{gathered}
$$

The index $\alpha$, appearing in the expressions for $\delta G_{\mu \nu}$ as well as in the varied equations of motion of the continuum, assumes values 2 and 3 . The expressions for $\delta T_{\mu \nu}$ and $2 \delta G_{\mu \nu}$ are given modulo factor $e^{i k x^{1}}$; this is, however, of no significance since we aim to equate them anyway.

When writing down the varied Einstein equations we can use formulas for the background solution to eliminate both $\rho_{0}$ and $\ddot{z}$ from our equations (and keep $z, \dot{z}, \varepsilon+\sigma, \lambda+\sigma$ and $\mu+\sigma$ only). Again, as in the background solution, we find that some terms of the varied Einstein equations are just time derivatives of other equations. In addition to the varied equations of motion of the continuum cited above, we obtain the following set of independent equations:

$$
\begin{gathered}
2 \dot{z} \dot{h}_{k k}+e^{-2 z} k^{2}\left(h_{k k}-h_{11}-4 \dot{z} h_{01}\right)=-\frac{1}{2} e^{-3 z}(\varepsilon+\sigma) h_{k k}, \\
\dot{h}_{11}-\dot{h}_{k k}=e^{-3 z}(\varepsilon+\sigma) h_{01}, \\
\dot{h}_{1 \alpha}-e^{-2 z} k^{2} h_{0 \alpha}=e^{-3 z}(\varepsilon+\sigma) h_{0 \alpha}, \\
\left(\ddot{h}_{22}-\ddot{h}_{33}\right)+3 \dot{z}\left(\dot{h}_{22}-\dot{h}_{33}\right)+e^{-2 z} k^{2}\left(h_{22}-h_{33}\right)=-e^{-3 z}(\mu+\sigma)\left(h_{22}-h_{33}\right), \\
\ddot{h}_{23}+3 \dot{z} \dot{h}_{23}+e^{-2 z} k^{2} h_{23}=-e^{-3 z}(\mu+\sigma) h_{23} .
\end{gathered}
$$

\subsection{Modes}

The set of equations we have found is evidently not totally coupled; it can be rather divided into several subsets of coupled equations. Physically, this means that there are different modes of wave propagation for the given wave covector. Since general relativity without matter and classical elasticity are both limit cases of relasticity, we expect that there will be two modes of gravitational waves, two modes of transversal acoustic waves and one mode of longitudal acoustic waves. Also, there should remain one odd mode coming from the freedom of choosing the zero time hypersurface.

First we have equations for $h_{22}-h_{33}$ and $h_{23}$, which can be written in a compact form as

$$
\ddot{h}_{i j}^{T}+3 \dot{z} \dot{h}_{i j}^{T}+\left[e^{-2 z} k^{2}+e^{-3 z}(\mu+\sigma)\right] h_{i j}^{T}=0,
$$


where $h_{i j}^{T}$ is the tensor part of $h_{i j}$ (a symmetric $3 \times 3$ matrix with nonzero components $h_{22}^{T}=$ $-h_{33}^{T}=\left(h_{22}-h_{33}\right) / 2$ and $\left.h_{23}^{T}=h_{23}\right)$. These equations clearly describe gravitational waves with polarizations $\oplus$ (the component $h_{22}^{T}$ ) and $\otimes$ (the component $h_{23}^{T}$ ); or, speaking in cosmological terms, the tensor perturbations (see [6]). The equations coincide with those valid in general relativity for an universe filled with an ideal fluid, see [10], if $\mu+\sigma$ vanishes. To see where this condition comes from, note that for an ideal fluid the energy per particle $\varepsilon$ depends only on the particle density $n$. If we write $n=\sqrt{\operatorname{det} H^{A B}}$, we can compute the second derivatives of $\varepsilon=\varepsilon(n)$ with respect to $H^{A B}$ and compare them with the expression in subsection 3.1. In this way we obtain $\mu=-n d \varepsilon / d n=(1 / 3) d \varepsilon / d z$. However, the identity for $d \varepsilon / d z$, to be found also in subsection 3.1, implies that the right hand side equals $-\sigma$. Thus, an ideal fluid can be defined as an elastic continuum with $\mu+\sigma=0$.

Next we have two pairs of equations containing $h_{0 \alpha}$ and $h_{1 \alpha}$ only,

$$
\begin{gathered}
\dot{h}_{0 \alpha}=\frac{2 \mu+3 \lambda+5 \sigma}{\varepsilon+\sigma} \dot{z} h_{0 \alpha}-\frac{\mu+\sigma}{\varepsilon+\sigma} h_{1 \alpha}, \\
\dot{h}_{1 \alpha}=\left[e^{-2 z} k^{2}+e^{-3 z}(\varepsilon+\sigma)\right] h_{0 \alpha} .
\end{gathered}
$$

Consider equations for $h_{02}$ and $h_{12}$. Since the perturbation of $g_{12}$ represents the shear strain in the $x^{2}$ direction and the perturbation of $g_{02}$ represents the motion of the particles of the continuum in the same direction (for an observer for whom the time $t$ is locally synchronized), this mode can be identified with the transversal acoustic wave that oscillates in the $x^{2}$ direction. Analogically, equations for $h_{03}$ and $h_{13}$ describe the propagation of a transversal acoustic wave that oscillates in the $x^{3}$ direction. These modes belong to the sector of vector cosmological perturbations.

There are three independent combinations of components of $h$ left, namely $h_{01}, h_{11}$ and $h_{k k}$, describing scalar cosmological perturbations. But they are all inflicted by the remaining freedom of choosing the zero time hypersurface. Pure shift of this hypersurface, corresponding to a plane wave with the wave covector $k=(k, 0,0)$, yields a contribution to $h$ proportional to $h_{01}=1$, $h_{11}=h_{22}=h_{33}=2 \dot{z}$. To identify physically relevant perturbations, we define combinations of components of $h$ that are invariant with respect to this hypersurface change. We do this by introducing $y_{01}, y_{11}$ and $y$ such that

$$
\begin{gathered}
h_{01}=y_{01}+y, \\
h_{11}=y_{11}+2 \dot{z} y, \\
h_{k k}=y_{11}+6 \dot{z} y .
\end{gathered}
$$

The interpretation of these formulas is as follows. If $\dot{z} \neq 0$, we can always choose the zero time hypersurface in such a way that $h_{k k}=h_{11}$. For such hypersurface, $y_{01}$ and $y_{11}$ are equal to $h_{01}$ and $h_{11}$ respectively. If we want to choose another hypersurface, we simply add the hypersurface shifting terms proportional to $y$. Since we still want $t$ to be the proper time of particles, we will have differential equation for $y$, but we can see that the physical information about the longitudal acoustic waves is contained in $y_{01}$ and $y_{11}$ only.

Using the definition of $y$ 's, we obtain a set of equations that can be neaten to one equation for $y$,

$$
\dot{y}=-\frac{1}{4 \dot{z}} e^{-3 z}(\varepsilon+\sigma) y_{01},
$$


and a pair of equations not containing $y$ which invariantly describe the propagation of a longitudal acoustic wave,

$$
\begin{gathered}
\dot{y}_{01}=\left[\frac{2 \mu+3 \lambda+5 \sigma}{\varepsilon+\sigma} \dot{z}+\frac{1}{4 \dot{z}} e^{-3 z}(\varepsilon+\sigma)\right] y_{01}-\frac{1}{2} \frac{\lambda+2 \mu+3 \sigma}{\varepsilon+\sigma} y_{11}, \\
\dot{y}_{11}=\left[2 e^{-2 z} k^{2}+\frac{3}{2} e^{-3 z}(\varepsilon+\sigma)\right] y_{01}-\frac{1}{4 \dot{z}} e^{-3 z}(\varepsilon+\sigma) y_{11}
\end{gathered}
$$

\section{Example}

Consider a continuum with the stiff ultrarigid state function introduced in [9]. The authors define the principal linear particle densities $n_{1}, n_{2}, n_{3}$ in terms of $H^{A B}$, and write the state function as

$$
\rho=A\left[\left(n_{1} n_{2}\right)^{2}+\left(n_{2} n_{3}\right)^{2}+\left(n_{3} n_{1}\right)^{2}\right]+B,
$$

where $A$ and $B$ are constants. If we choose, for simplicity, $A=1$ and $B=0$, the state function as a function of $H^{A B}$ is

$$
\varrho=\frac{1}{2}\left(H^{C C} H^{D D}-H^{C D} H^{C D}\right) .
$$

If we treat all $H^{A B}$ 's as truly independent variables, the second term depends on the antisymmetric part of $H^{A B}$; however, there is no need to modify it as long as we use our formalism of symmetrized higher derivatives.

The first and second derivatives of $\varrho$ with respect to $H^{A B}$ are

$$
\begin{aligned}
& \frac{\partial \varrho}{\partial H^{A B}}=\delta_{A B} H^{C C}-\delta_{A C} \delta_{B D} H^{C D}, \\
& \frac{\partial^{2} \varrho}{\partial H^{A B} \partial H^{C D}}=\delta_{A B} \delta_{C D}-\delta_{A C} \delta_{B D} .
\end{aligned}
$$

Evaluating $\varrho$ and its derivatives for $H^{A B}=e^{-2 z} \delta^{A B}$ we get

$$
\varrho=3 e^{-4 z}, \quad \frac{\partial \varrho}{\partial H^{A B}}=2 e^{-2 z} \delta_{A B}, \quad \frac{\partial^{2} \varrho}{\partial H^{A B} \partial H^{C D}}=\delta_{A B} \delta_{C D}-\delta_{A C} \delta_{B D},
$$

and, consequently,

$$
\rho_{0}=0, \quad \varepsilon=3 e^{-z}, \quad \sigma=e^{-z}, \quad \lambda=-e^{-z}, \quad \mu=e^{-z} .
$$

The equation $6 \dot{z}^{2}=\rho_{0}+e^{-3 z} \varepsilon$ reads

$$
\dot{z}^{2}=\frac{1}{2} e^{-4 z}
$$

and has an (expanding) solution

$$
z=\frac{1}{4} \ln 2+\frac{1}{2} \ln t
$$

This is just the well known formula $a \propto \sqrt{t}$ for a universe filled with an ideal fluid with the equation of state $\sigma=(1 / 3) \varepsilon$ (or, in more common notations, $p=(1 / 3) \rho$ ).

Using $\dot{z}=(2 t)^{-1}$ and $e^{-2 z}=(\sqrt{2} t)^{-1}$, we can express coefficients in the equation, or system of equations, for each kind of waves as functions of time. The equation for gravitational waves 
reads

$$
\ddot{h}_{i j}^{T}+\frac{3}{2 t} \dot{h}_{i j}^{T}+\left(\frac{k^{2}}{\sqrt{2} t}+\frac{1}{t^{2}}\right) h_{i j}^{T}=0 .
$$

Besides of that, we have a pair of equations for transversal acoustic waves,

$$
\dot{h}_{0 \alpha}=\frac{1}{2 t} h_{0 \alpha}-\frac{1}{2} h_{1 \alpha}, \quad \dot{h}_{1 \alpha}=\left(\frac{k^{2}}{\sqrt{2} t}+\frac{2}{t^{2}}\right) h_{0 \alpha},
$$

and a triplet of equations for longitudal acoustic waves,

$$
\begin{gathered}
\dot{y}=-\frac{2}{t} y_{01}, \\
\dot{y}_{01}=\frac{3}{2 t} y_{01}-\frac{1}{2} y_{11}, \quad \dot{y}_{11}=\left(\frac{\sqrt{2}}{t} k^{2}+\frac{3}{t^{2}}\right) y_{01}-\frac{1}{t} y_{11} .
\end{gathered}
$$

The first-order equations for $h_{0 \alpha}$ and $h_{1 \alpha}$, as well as those for $y_{01}$ and $y_{11}$, can be combined into a second-order equation for either variable. We choose the variables $h_{0 \alpha}$ and $y_{01}$, because the corresponding equations contain $k^{2}$ only as a factor in front of the variable itself, thus becoming hyperbolic partial differential equations when one passes from $k^{2}$ back to the Laplacian. The equations read

$$
\begin{gathered}
\ddot{h}_{0 \alpha}-\frac{1}{2 t} \dot{h}_{0 \alpha}+\left(\frac{k^{2}}{2 \sqrt{2} t}+\frac{3}{2 t^{2}}\right) h_{0 \alpha}=0, \\
\ddot{y}_{01}-\frac{1}{2 t} \dot{y}_{01}+\left(\frac{k^{2}}{\sqrt{2} t}+\frac{3}{2 t^{2}}\right) y_{01}=0 .
\end{gathered}
$$

A remarkable feature of these equations is that they coincide in the limit of small $k$. Thus, if we use for the description of longitudinal and transversal acoustic waves the functions $h_{0 \alpha}$ and $y_{01}$, the waves behave identically in the regime in which they stay well outside the horizon. There is no point in extending the comparison to gravitational waves, since the field $h_{i j}^{T}$ in the corresponding equation is of completely different nature than the fields $h_{0 \alpha}$ and $y_{01}$ in the equations for acoustic waves (which are at least akin). Note, however, that if we pick the variables $h_{1 \alpha}$ and $y_{11}$ instead of $h_{0 \alpha}$ and $y_{01}$ to describe acoustic waves, or multiply $h_{0 \alpha}$ and $y_{01}$ by $e^{-2 z}$ (the same factor that appears in the definition of $h_{i j}$ in terms of $\delta g_{i j}$ ), the resulting equations will coincide not only with each other, but also with the equation for $h_{i j}^{T}$ in the limit of small $k$.

For both gravitational and acoustic waves, oscillating fields as functions of time are depicted in figure 1. The curves denoted by $g$ ('gravitational'), $t$ ('transversal') and $l$ ('longitudinal') are graphs of the functions $h_{22}^{T}$ or $h_{23}^{T}, h_{02}$ or $h_{03}$, and $y_{01}$ respectively. For comparison, graphs of the functions $h_{22}^{T}$ or $h_{23}^{T}$ and $y_{01}$ for an ideal fluid with the equation of state $\sigma=(1 / 3) \varepsilon$ are included into the figure, too (denoted by $g_{\text {fluid }}$ and $l_{\text {fluid }}$; of course, there are no transversal acoustic waves in an ideal fluid). All curves are computed for $k=1$, with the oscillating field equal to 1 and its derivative vanishing at the moment $t=0.01$. The waves cross the horizon at $t \sim 1$; before that their wavelength is larger and afterwards it is smaller than the horizon length. The main new effect of nonzero shear stresses, distinguishing the continuum under consideration from an ideal fluid, are oscillations of the fields at superhorizon scales. Asymptotically, the oscillating factor is $\cos [(\sqrt{15} / 4) \log t+\psi]$ for all three kinds of waves. 


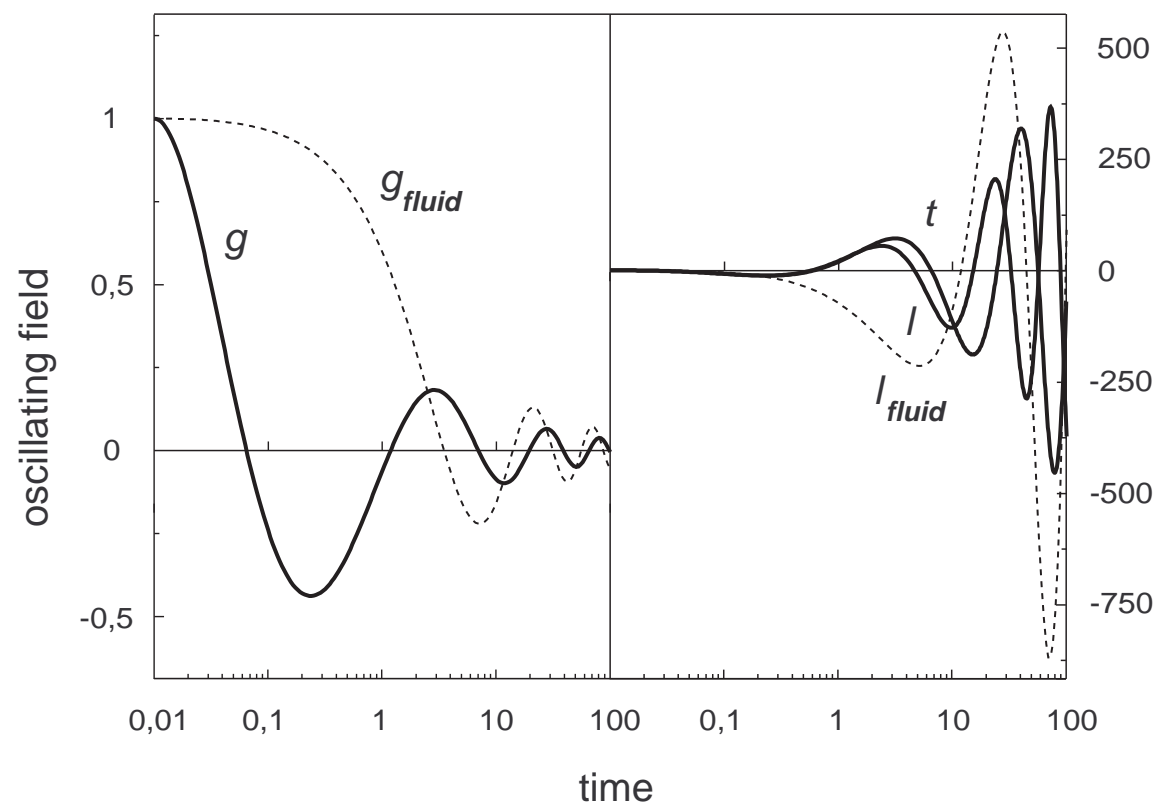

Figure 1: Time evolution of gravitational and acoustic waves

\section{Conclusion}

We have developed a formalism describing the propagation of plane waves in a general relativistic homogeneous and isotropic flat continuum. In comparison with [5], where the same problem is studied, we did not include into the theory small perturbations to the Euclidean metric of the material manifold (tensor $b_{a b}$ in the notation of [5]). In this way we simplified the matters as much as possible.

We were successful in identifying relativistic generalizations of acoustic waves. The longitudinal mode was encumbered with the 'time wave' gauge freedom, and the definition of gauge invariant $y$ 's was needed. After introducing them we were able to describe the wave by two coupled first-order differential equations. This is equivalent to one second-order equation, hence our theory is a straightforward generalization of its nonrelativistic counterpart. Again, this can be compared with [5] where the propagation of the longitudinal wave is described by two coupled second-order equations (equations (3.12) and (3.15) in subsection 3.1). The cited work differs from ours in that respect that it uses Newtonian gauge. Of course, the transition from one gauge to another cannot increase the number of degrees of freedom, therefore in Newtonian gauge the longitudinal wave must be also described by two first-order equations (imposing constraints on initial conditions for the second-order equations). Perhaps the comoving proper-time gauge we have chosen for our calculations is better adapted for derivation of these more fundamental equations.

We kept the formalism as easy and simple as possible, although sometimes we have preferred comprehensibility to simplicity. For example, the introduction of $\rho_{0}$ and $\varepsilon$ was not necessary. We could use $\varrho$ (and scalars entering the derivatives of $\varrho$ with respect to $H^{A B}$ ) only, but we felt that the quantities $\varepsilon$ (and $\sigma, \mu$ and $\lambda$ ) were more appropriate for the description of the continuum. 
Also, we introduced $z$ and $h$ 's just to simplify the expressions even if the meaning of $h_{0 i}$ is far from obvious. And our choice of $y$ 's reflected the most natural initial condition $h_{11}=h_{k k}$. On the other hand, the fact that the resulting equations do have singularity for $\dot{z}=0$ suggests that this condition is not the best. For those interested in nonsingular equations, note that using

$$
\begin{gathered}
h_{01}=v_{01}+v \\
h_{k k}=v_{k k}+6 \dot{z} v \\
h_{11}=\frac{4 e^{-2 z} k^{2}+2 e^{-3 z}(\varepsilon+\sigma)}{4 e^{-2 z} k^{2}} v_{k k}+2 \dot{z} v
\end{gathered}
$$

we obtain equations

$$
\begin{gathered}
\dot{v}=-\frac{e^{-3 z}(\varepsilon+2 \mu+3 \lambda+6 \sigma)}{4 e^{-2 z} k^{2}+3 e^{-3 z}(\varepsilon+\sigma)} v_{k k}, \\
\dot{v}_{k k}=\frac{6 e^{-3 z}(\varepsilon+2 \mu+3 \lambda+6 \sigma)}{4 e^{-2 z} k^{2}+3 e^{-3 z}(\varepsilon+\sigma)} \dot{z} v_{k k}+2 e^{-2 z} k^{2} v_{01}, \\
\dot{v}_{01}=\frac{2 \mu+3 \lambda+5 \sigma}{\varepsilon+\sigma} \dot{z} v_{01}-\left[\frac{1}{2} \frac{2 \mu+\lambda+3 \sigma}{\varepsilon+\sigma}+\frac{2 e^{-3 z}(\mu+\sigma)}{4 e^{-2 z} k^{2}}-\frac{e^{-3 z}(\varepsilon+2 \mu+3 \lambda+6 \sigma)}{4 e^{-2 z} k^{2}+3 e^{-3 z}(\varepsilon+\sigma)}\right] v_{k k} .
\end{gathered}
$$

However, these equations look no simpler than the equations for $h$ 's, and we used $\dot{z} \neq 0$ when deriving them anyway.

When applying our theory to a stiff ultrarigid continuum, we found that the time evolution of longitudinal and transversal acoustic waves, if described by the functions $h_{0 \alpha}$ and $y_{01}$, coincide in the limit of small $k$. This is not a unique feature of the state function considered. The same statement holds for any 'linear' continuum, that is, continuum with $\rho_{0}=0$ and $\sigma, \lambda$ and $\mu$ proportional to $\varepsilon$, provided the constraint on the coefficients of proportionality following from the relation $d \sigma / d z=-(2 \sigma+3 \lambda+2 \mu)$ is satisfied.

Acknowledgement. This work was supported by the grant VEGA 1/3042/06.

\section{References}

[1] M. Karlovini, L. Samuelsson, Class.Quant.Grav. 20 (2003) 3613; gr-qc/0211026 v2.

[2] B. Carter, H. Quintana, Proc. Roy. Soc. Lond. A331 (1972) 57.

[3] L. D. Landau, E. M. Lifshitz: Theory of Elasticity, Pergamon, New York (1986).

[4] B. Carter, H. Quintana, Phys.Rev. D16 (1977) 2928.

[5] M. Bucher, D. N. Spergel, Phys.Rev. D60 (1999) 043505; astro-ph/9812022.

[6] A. R. Liddle, D. H. Lyth: Cosmological inflation and large-scale structure, Cambridge University Press (2000).

[7] R. Beig, B. G. Schmidt, Class.Quant.Grav. 20, (2003) 889; gr-qc/0211054 v2.

[8] M. Fecko: Differential Geometry and Lie Groups for Physicists, Cambridge University Press (2006).

[9] M. Karlovini, L. Samuelsson, Class.Quant.Grav. 21 (2004) 4531; gr-qc/0401115.

[10] L. D. Landau, E. M. Lifshitz: The Classical Theory of Fields, Pergamon, New York (1975). 